\title{
Fatty metamorphosis and other patterns in fibrous dysplasia
} Vinod B Shidham*1, Ashwini Chavan¹, R Nagarjun Rao1, Richard A Komorowski ${ }^{1}$ and Zeenath Asma ${ }^{1,2}$

\author{
Address: ${ }^{1}$ Dept. of Pathology, Medical College of Wisconsin, Milwaukee, WI, USA and ${ }^{2}$ Pathology at Metropolitan Group Hospital Program, \\ Chicago, IL, USA \\ Email: Vinod B Shidham* - vshidham@mcw.edu; Ashwini Chavan - achavan@mcw.edu; R Nagarjun Rao - arao@mcw.edu; \\ Richard A Komorowski - rkomor@mcw.edu; Zeenath Asma - zasmapath2002@yahoo.com \\ * Corresponding author
}

Published: 28 August 2003

BMC Musculoskeletal Disorders 2003, 4:20
Received: 03 June 2003

Accepted: 28 August 2003

This article is available from: http://www.biomedcentral.com/I47/-2474/4/20

(C) 2003 Shidham et al; licensee BioMed Central Ltd. This is an Open Access article: verbatim copying and redistribution of this article are permitted in all media for any purpose, provided this notice is preserved along with the article's original URL.

\begin{abstract}
Background: Interpretation of small biopsy fragments from suspected lesions of fibrous dysplasia with unusual clinical and / or radiological features may be challenging due to wide histomorphological spectrum of stromal appearances. Awareness of these variations should improve diagnostic confidence.

Methods: We retrospectively studied 26 cases of fibrous dysplasia (F- 19, M- 7; Ages ranged from 10 to 53 years) with confirmed diagnosis. The sites of the lesions were skull bones (9), humerus (I), femur (8), tibia (2), fibula (3), talus (I), mandible (I), and maxilla (I).

Results: Different stromal patterns, variably admixed with the classical pattern, were observed in $58 \%(15 / 26)$ of the cases. $20 \%(3 / 15)$ of these had more than one pattern. Focal fatty metamorphosis as groups of fat cells in the central portion of the lesion in the stroma of fibrous dysplasia between osseous trabeculae was observed in $23 \%(6 / 26)$ cases. Other patterns included myxoid stroma in $16 \%(4 / 26)$, collagenization of stroma in $12 \%(3 / 26)$, stroma rich pattern (with paucity of trabeculae) in $12 \%(3 / 26)$, foci of few foam cells in $23 \%(6 / 26)$, and calcified spherules in $12 \%(3 / 26)$. Focal osteoblastic rimming of trabeculae was observed only in $4 \%(1 / 26)$.
\end{abstract}

Conclusions: Various stromal variations and previously unreported fatty metamorphosis were frequently observed in fibrous dysplasia.

\section{Background}

Most of the lesions of fibrous dysplasia are asymptomatic and are usually detected incidentally on roentgenograms. It may manifest as pathologic fracture of weight bearing bones or may present as swelling [1,2]. The defect is usually in the form of a well-defined zone of rarefaction with fine granular homogenous appearance simulating ground glass pattern and often surrounded by a narrow rim of sclerotic bone [3].

The lesions of fibrous dysplasia may occur in diaphysis, metaphysis, and very rarely in epiphysis. Ribs, jawbones, skull bones, femur, and tibia are commonly involved. Spine is usually spared. The disease may be monostotic or polyostotic. It may also be a component of McCune- 
Albright syndrome characterized by a triad of polyostotic fibrous dysplasia, localized café-au-lait hyperpigmentation of the skin, and hyperfunctional endocrinopathies such as precocious puberty [2].

Monostotic fibrous dysplasia, polyostotic fibrous dysplasia, and McCune-Albright syndrome dysplasia have the mutation of one of the cell surface receptors, G protein, associated with a variety of important cell functions controlled through effector system that generates intracellular second messengers. The mutation involves ligand-independent, alpha-subunit of stimulatory $G$ protein (GNAS1) leading to its activation. Two specific mutations have been reported: $\mathrm{C}$ to $\mathrm{T}$ mutation resulting in Arg201Cys and G to A mutation resulting in Arg201His. They are located near the site interacting with gama-phosphate of GTP leading to interference with its hydrolysis to GDP $[4,5]$.

Occasionally, large expansile mass lesions may bulge into the adjacent soft tissue. Some lesions with significant cartilage components may show ring-like or dot-like calcifications. Others may produce aggressive appearing radiologic features, suggestive of sarcoma, leading to biopsy and surgical pathology evaluation. When submitted as small biopsy fragments, these may be difficult to interpret. The biopsy fragments may be from areas predominantly showing variant histomorphological patterns of fibrous dysplasia.

We observed foci of previously unreported focal fatty metamorphosis within stroma that occupied inter-trabecular areas in the biopsy fragments retrieved from the central portion of the lesion away from the periphery [6]. Lack of awareness of this and other histomorphological variations may compromise interpretation, especially when biopsy is submitted as small tissue fragments and for frozen sectioning. To study the morphological spectrum in general, and fatty metamorphosis in particular, we retrospectively evaluated cases with confirmed diagnosis of fibrous dysplasia.

\section{Methods}

We analyzed the histomorphological spectrum of fibrous dysplasia in 26 cases (F- 19, M- 7; Ages ranged from 10 to 53 years) with confirmed diagnosis based on clinical, radiological, and pathological data. The sites of the lesions included skull bones (9), humerus (1), femur (8), tibia (2), fibula (3), talus (1), mandible (1), and maxilla (1). Different histomorphological patterns were recognized. Focal fatty metamorphosis was defined as presence of groups of fat cells in the biopsy fragments from the lesion in inter-trabecular areas between osseous trabeculae without osteoblastic lining. Other patterns were similar to those described in the literature $[1-3,7]$.

\section{Results}

Different stromal patterns, variably admixed with the classical pattern (Figure 1), were observed in 58\%(15/26) of the cases. $20 \%(3 / 15)$ of these had more than one pattern. The spectrum of histomorphological patterns observed included focal fatty metamorphosis (Figure 2) in 23\%(6/ $26)$, collagenization of stroma (Figure 3 ) in 12\%(3/26), stroma rich pattern (with paucity of trabeculae, Figure 4) in $12 \%(3 / 26)$, and myxoid stroma (Figure 5) in $16 \%(4 /$ 26). Focal osteoblastic rimming of trabeculae was observed in $4 \%(1 / 26)$. Focal collections of few foam cells were seen in $23 \%(6 / 26)$ cases. Osseous component as calcified spherules was observed in 3 cases (Figure 2C).

\section{Discussion}

Fibrous dysplasia results from aberrant development of bone leading to single or multiple skeletal defects. The lesions are usually seen as expansile medullary defects with thinning of the cortex. They show fibro-osseous metaplasia with abundance of osseous trabeculae intermingled with stroma consisting of proliferating fibroblasts producing dense collagenous matrix $[1,2,8]$. The spindle cells tend to be plump but do not show cytologic atypia. The osseous trabeculae of woven bone present in the stroma typically do not have osteoblastic rimming [8].

The stroma and osseous trabeculae may show variations from the classical histomorphological pattern leading to interpretation challenge, especially with small biopsy fragments retrieved from lesions with unusual clinical and radiological features simulating bone neoplasms. The characteristic fibro-osseous metaplasia seen in fibrous dysplasia may resemble patterns associated with lowgrade parosteal osteosarcoma and fibrous dysplasia - like low-grade intramedullary osteosarcoma [8].

Awareness of the histomorphological spectrum of fibrous dysplasia would facilitate correct interpretation. Many of the variations in the histomorphological spectrum have been described in the literature $[1,2,8]$. In this study, the commonest pattern observed in association with the classical pattern was fatty metamorphosis (figure 2) in the form of collection of adipocytes. This pattern observed in $23 \%(6 / 26)$ of our cases has not been reported previously. This feature was distinct from the collection of foam cells reported in the literature $[1,2,8]$.

Fatty metamorphosis (Figure 2) was observed adjacent to areas showing classical pattern of fibrous dysplasia. Adipocytes were intermingled in variable proportions with fibroblastic stroma. Some areas showed predominantly adipocytes with scant fibroblastic component (figure 2, arrow ' $a$ '), and other areas showed predominantly fibrous stroma with occasional adipocytes (figure 2, arrow 'b'). In the stroma, the osseous trabeculae were usually small and 


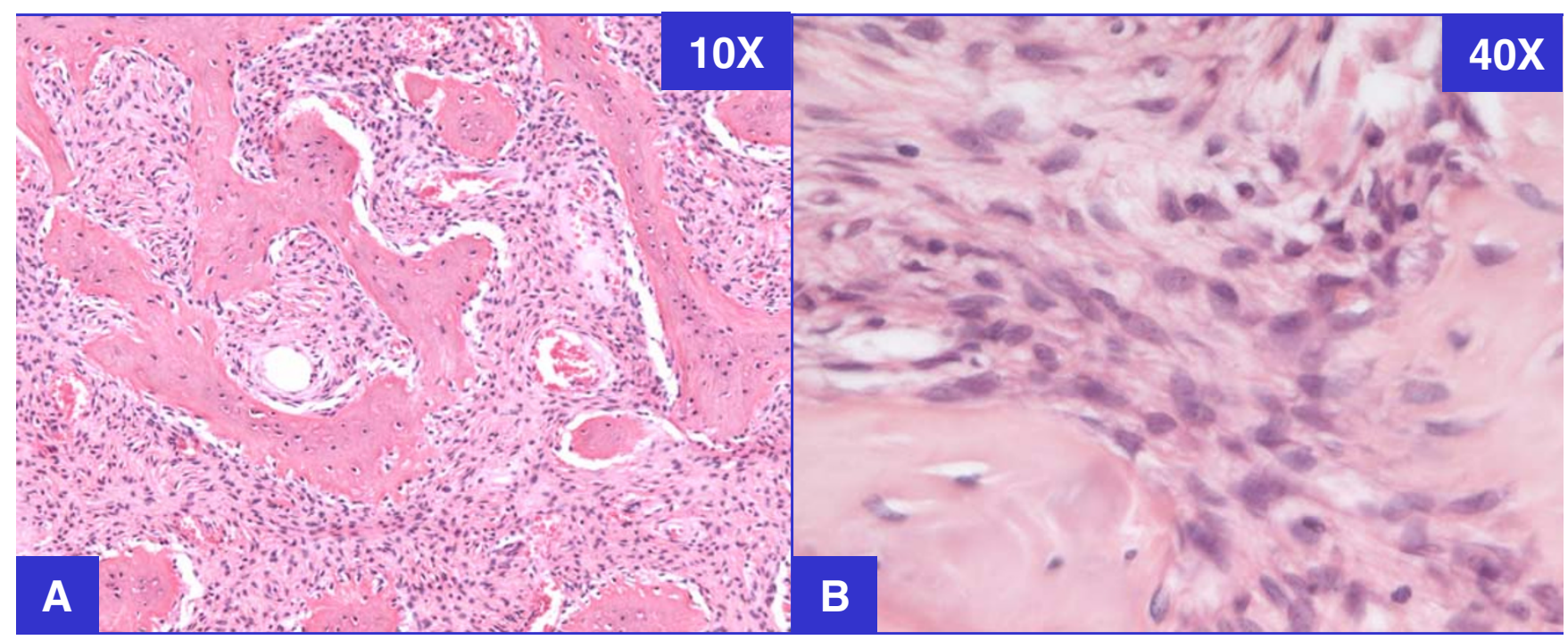

\section{Figure I}

Fibrous dysplasia with classical pattern. A \& B. The sections show fibroblastic stroma with plump spindle cells interspersed with osseous trabeculae without osteoblastic rimming (A \& B- H\&E).

ossicle-like. Trilineage hematopoietic elements were not observed in the areas showing fatty metamorphosis. This was useful adjunct to rule out the adipocytes from bone marrow component.

When fatty metamorphosis is the predominant pattern, it may be interpreted as non-representative of fibrous dysplasia and may be regarded as insufficient for diagnosis. In fact, this problem was faced initially in one of our cases with tiny fragments of biopsy with predominant fatty metamorphosis pattern (Figure 2). Absence of trilineage marrow and presence of osseous trabeculae without osteoblastic rimming would favor fibrous dysplasia (Figure 2).

It is interesting to correlate that fat metabolism is partly regulated by epinephrine receptors which signal through
GNAS1 protein. Nonfunctional mutations result in fat accumulation. However, functional mutations observed in fibrous dysplasia, have been associated with uncontrolled fat production in thyroid tumor cells. Thus, it is possible that focal fatty metamorphosis in fibrous dysplasia observed in this study may be related to GNAS1 mutations.

Other stromal patterns were variably admixed with the classical pattern. They were observed in 58\%(15/26) of the cases and $20 \%(3 / 15)$ had more than one pattern. The spectrum included focal fatty metamorphosis (Figure 2) in $23 \%(6 / 26)$, collagenization of stroma (Figure 3 ) in $12 \%(3 / 26)$, stroma rich pattern (with paucity of trabeculae, Figure 4 ) in $12 \%(3 / 26)$, and myxoid stroma (Figure 5 ) in $16 \%(4 / 26)$. Osteoblastic rimming of trabeculae was rare and was observed only focally in one case $(4 \%, 1 / 26)$. 


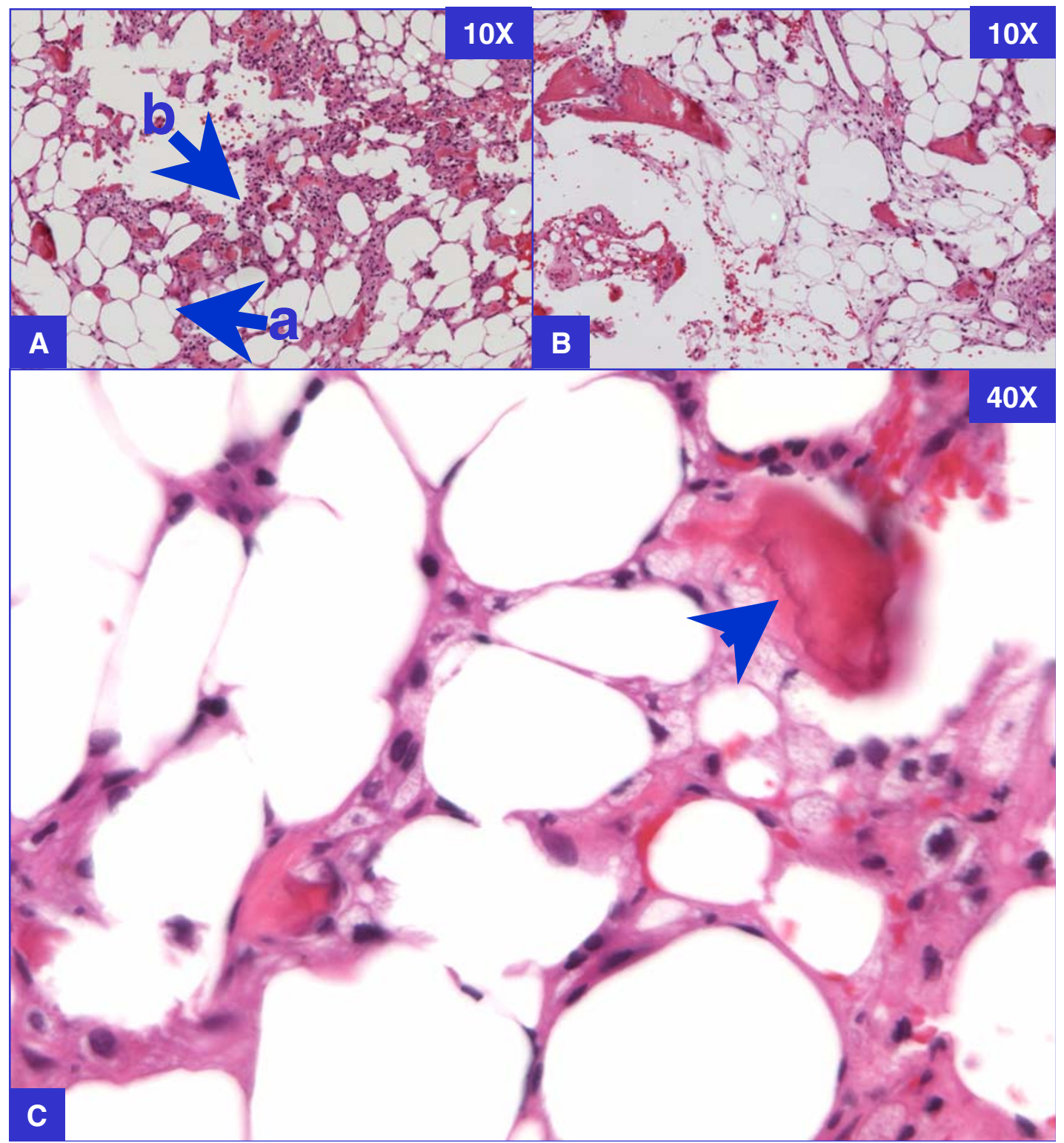

Figure 2

Fibrous dysplasia with fatty metamorphosis. A. \& B. Sections show the stroma of fibrous dysplasia with significant number of adipocytes interspersed between the fibroblastic stroma cells. Arrow 'a' shows relatively larger number of adipocytes and arrow 'b' shows relatively small number of adipocytes among the fibroblastic stroma of fibrous dysplasia. C. Few small ossiclelike osseous trabeculae without osteoblastic rimming are present (arrow head) (A, B, \& C- H\&E). 


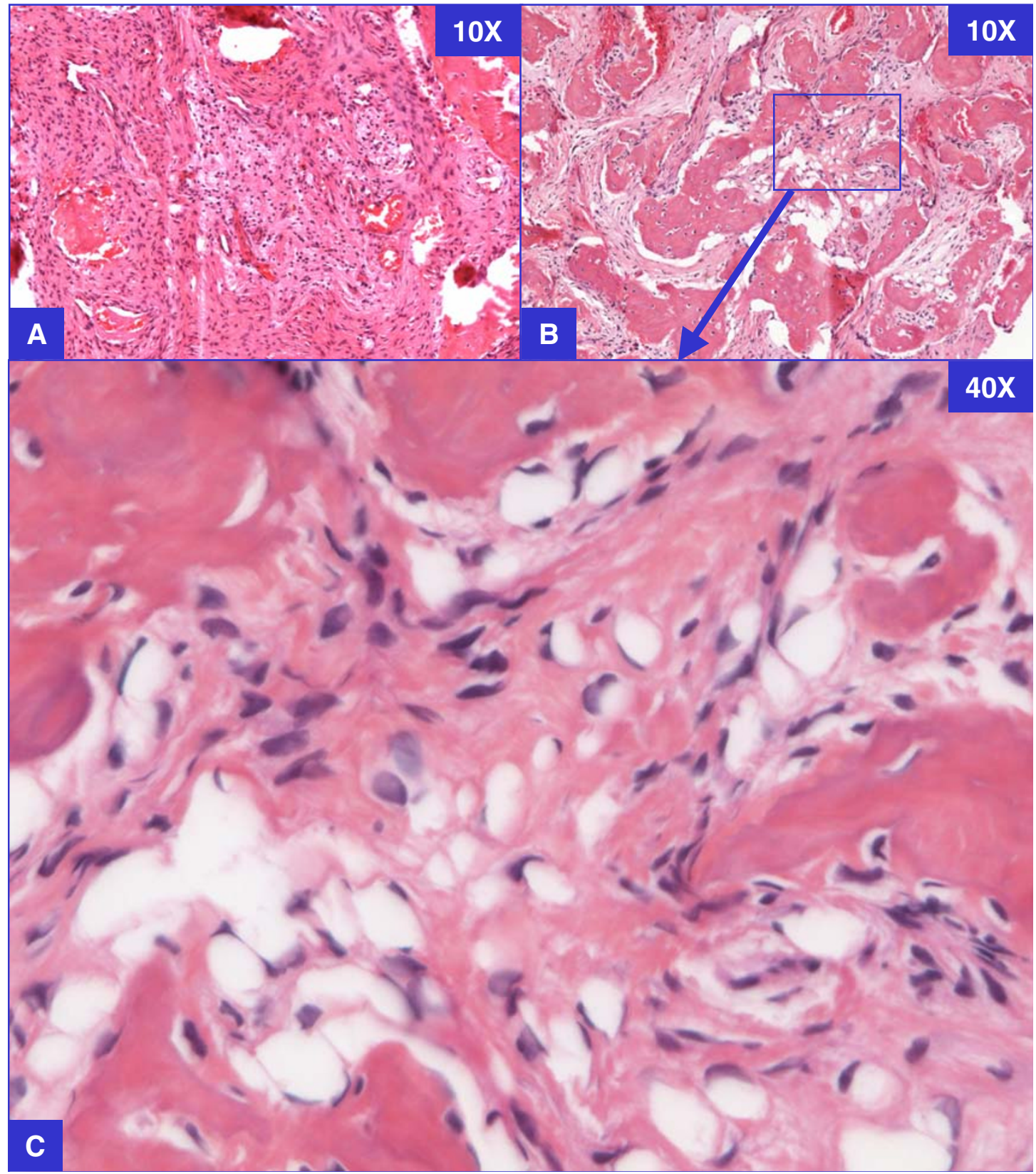

\section{Figure 3}

Fibrous dysplasia with collagenized stroma. The cells in the stroma are relatively less plump with significant portion of intercellular collagen. The osseous trabeculae without osteoblastic rimming are present amongst this stroma. This case also shows additional fatty metamorphosis pattern. 


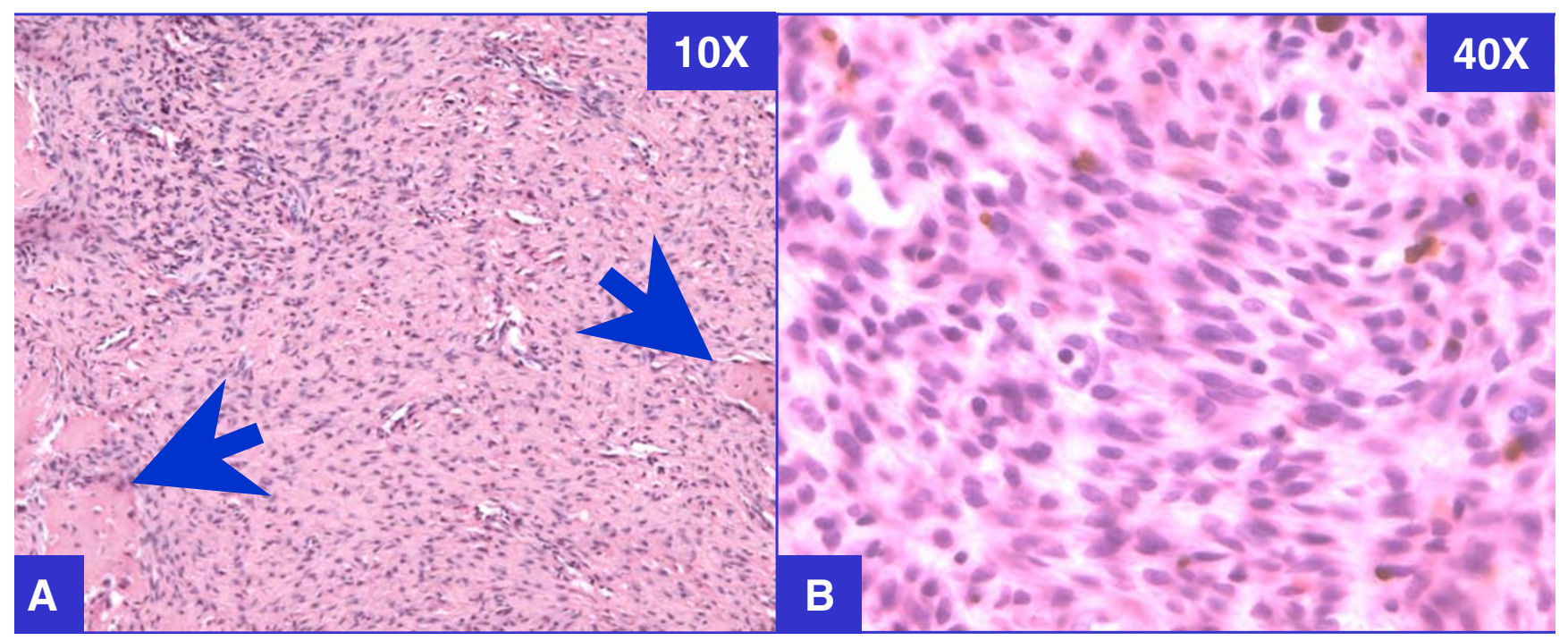

Figure 4

A \& B. Fibrous dysplasia with paucitrabecular pattern with hypercellular stroma showing plump cells. The osseous trabeculae are widely spaced (arrows). (A \& B- H\&E)

Focal collections of few foam cells were seen in 23\% (6/ 26) cases; however, they were not present as a significantly prominent component. Osseous component as calcified spherules were observed in 3 cases $[2,9]$ and were associated with fatty metamorphosis in one case (Figure 2C).

These variations in the pattern of stroma in fibrous dysplasia have been described in the literature, however; their frequency has not been documented $[1,2,8]$. These patterns were observed in addition to the classical pattern in all $58 \%$ of the cases. Three cases showed more than one pattern. Surprisingly fatty metamorphosis pattern was the commonest pattern $(23 \%, 6 / 26)$. This has not been highlighted previously in the literature. Islands of cartilage, reported in up to $10 \%$ of cases $[1,2]$ were not observed in this study. This may be due to the small size of biopsy fragments in most of our cases.
Focal osteoblastic rimming along the osseous trabeculae was seen in rare cases $(4 \%, 1 / 26)$. Lack of osteoblastic rimming is of importance in differentiating fibrous dysplasia from benign entities such as callus, osteoblastoma, ossifying fibroma, osteofibrous dysplasia, and some low-grade neoplasms such as low-grade osteosarcoma, low-grade intramedullary fibrous dysplasia like osteosarcoma, and parosteal osteosarcoma [8].

\section{Conclusions}

We observed foci of previously unreported fatty metamorphosis in this retrospective study of a series of fibrous dysplasia cases. In addition other stromal patterns were also observed in significant proportion. Focal osteoblastic rimming was seen rarely. Awareness of fatty metamorphosis and other stromal patterns should improve diagnostic 


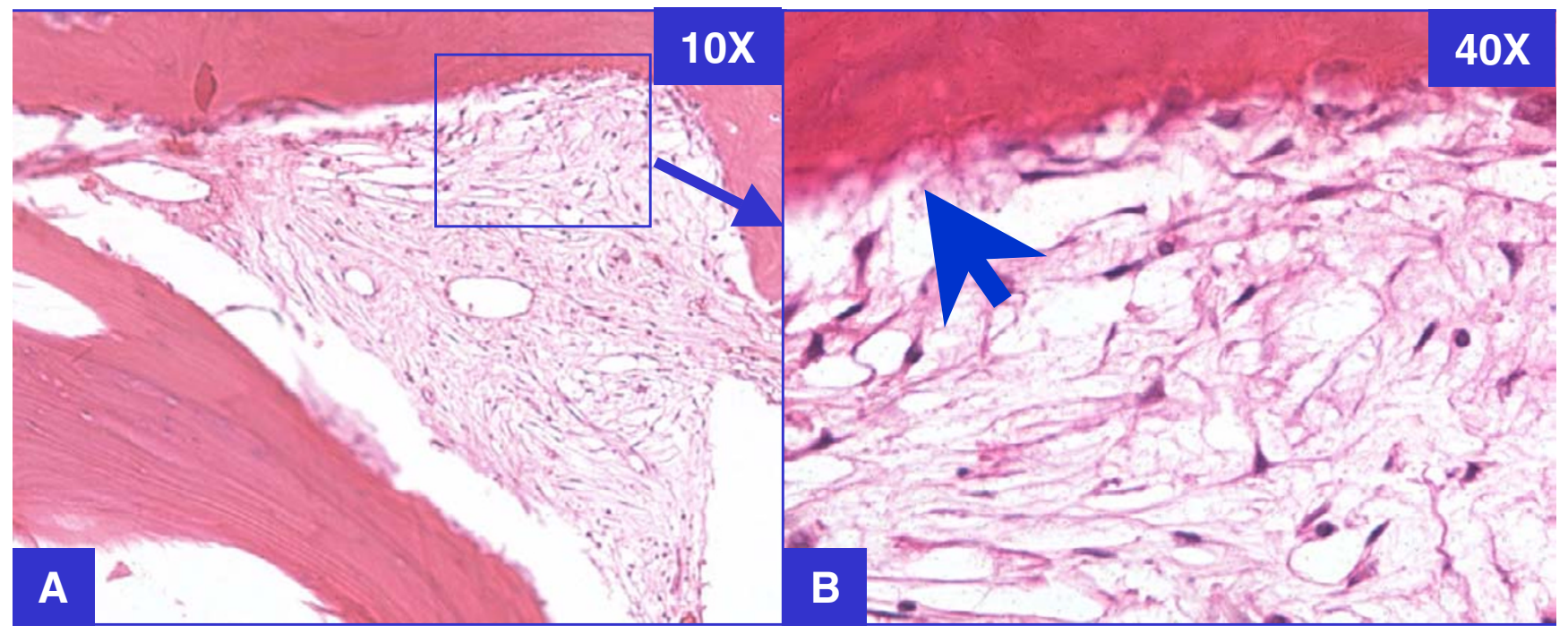

Figure 5

Fibrous dysplasia with myxoid stroma. A\&B. The stroma is myxoid with presence of few scattered stellate shaped cells. B. The osseous trabeculae lack the osteoblastic rimming (arrow). (A\&B- H\&E)

confidence in interpreting small biopsies of bone lesions suspicious for fibrous dysplasia.

\section{Competing interests}

None declared.

\section{Authors' contributions}

VS conceived, designed, and carried out the entire study in addition to the drafting of manuscript. ZA participated in the design of the study and assisted in data collection. AC, RNR, and RK all participated in its design and coordination.

\section{Acknowledgements}

We thank K. Krishnan Unni, MBBS for critical review of the manuscript with important suggestions for the improvement. We also thank Patsy Gill for the secretarial support.

\section{References}

I. Unni KK: Conditions that simulate bone neoplasms. Dahlin's Bone Tumors. General Aspects and Data on I I,087 Cases Fifthth edition. Edited by: Unni KK. Philadelphia, Lippincott-Raven; 1996:367-376.

2. Vigorita VS: Orthopedic Pathology. Philadelphia, Lippincott Williams and Wilkins 1999:300-307.

3. Anand MKN: Fibrous Dysplasia. eMedicine Specialties > Radiology > Musculoskeletal [http://www.emedicine.com/radio/topic284.htm].

4. Weinstein LS, Shenker A, Gejman PV, Merino MJ, Friedman E and Spiegel AM: Activating mutations of the stimulatory $\mathbf{G}$ protein in the McCune-Albright syndrome. N Engl J Med 1991, 325:1688-1695.

5. Cohen MM Jr: Fibrous dysplasia is a neoplasm. Am J Med Genet 2001, 98:290-293.

6. Asma Z, Dimitrov R, Chavan A, Rao NR, Komorowski RA and Shidham VB: Fatty metamorphosis and other stromal variations in fibrous dysplasia. Modern Pathology 2002, I 5: I A-368A. Abstract no. 28

7. Clayer C: Fibrous Dysplasia. eMedicine Specialties > Orthopedic Surgery > Neoplasms [http://www.emedicine.com/orthoped/ topic487.htm].

8. Mirra JM, Gold RH and Picci P: Osseous tumors of intramedullary origin. In Bone Tumors Edited by: Mirra JM, Picci P, Gold RH. Philadelphia, Lea \& Febiger; 1989:|43-438. 
9. Voytek TM, Ro JY, Edeiken J and Ayala AG: Fibrous dysplasia and cemento-ossifying fibroma. A histologic spectrum. Am J Surg Pathol 1995, 19:775-78I.

\section{Pre-publication history}

The pre-publication history for this paper can be accessed here:

http://www.biomedcentral.com/1471-2474/4/20/prepub

Publish with Bio Med Central and every scientist can read your work free of charge

"BioMed Central will be the most significant development for disseminating the results of biomedical research in our lifetime. " Sir Paul Nurse, Cancer Research UK

Your research papers will be:

- available free of charge to the entire biomedical community

- peer reviewed and published immediately upon acceptance

- cited in PubMed and archived on PubMed Central

- yours - you keep the copyright 